\section{La calidad de los servicios de radiología en cinco países latinoamericanos}

\author{
Ileana Fleitas, ${ }^{1}$ \\ Carlos C. Caspani, ${ }^{2}$ Cari \\ Borrás, ${ }^{1}$ Maria-Cristina Plazas, ${ }^{3}$ \\ Alberto A. Miranda, ${ }^{4}$ \\ Maria-Ester Brandan ${ }^{5}$ \\ y Roxana de la Mora 6
}

Forma de citar: Fleitas I, Caspani CC, Borrás C, Plazas MC, Miranda AA, Brandan ME, et al. La calidad de los servicios de radiología en cinco países latinoamericanos. Rev Panam Salud Publica. 2006;20(2/3):113-24.

Palabras clave: radiología, servicio de radiología en hospital, radiografía torácica, mamografía, fluoroscopia, control de la calidad, América Latina.

\footnotetext{
1 Organización Panamericana de la Salud, Washington, D.C., Estados Unidos de América. La correspondencia se debe enviar a Ileana Fleitas, PAHO/WHO, 525 Twenty-third Street, NW, Washington, D.C. 20037, United States of America. Correo electrónico: ileana@cceem. sld.cu

2 Radiofísica Sanitaria (RFSF), Secretaría de Salud de Santa Fe, Santa $\mathrm{Fe}$, Argentina.

3 Instituto Nacional de Cancerología (INC), Ministerio de Salud, Santa Fe de Bogotá, Colombia.

4 Instituto Boliviano de Ciencia y Tecnología Nuclear (IBTEN), Ministerio de Desarrollo Sostenible y Medio Ambiente, La Paz, Bolivia.

5 Instituto de Física, Universidad Nacional Autónoma de México, México, D.F., México.

6 Centro de Control Estatal de Equipos Médicos, Ministerio de Salud Pública, Ciudad de La Habana, Cuba.
}

\section{RESUMEN}

Objetivo. Determinar la correlación entre ciertos indicadores de calidad para los servicios de imaginología y la certeza en la interpretación de los exámenes radiológicos para cuatro quejas frecuentes: las masas de la mama, el malestar del aparato digestivo, el dolor de espalda y los sintomas de la tuberculosis.

Métodos. Se evaluaron veintiséis servicios de radiología en Argentina, Bolivia, Colombia, Cuba y México. Se evaluaron los equipos de mamografía y de radiografíalfluoroscopia convencional usados en los servicios seleccionados utilizando protocolos comunes, hojas de especificaciones técnicas, instrumentos de prueba, maniquies y sistemas de dosimetría calibrados. Los estudios se realizaron en establecimientos de complejidad media. Se obtuvo el consentimiento informado de todos los pacientes estudiados, y se garantizó la confidencialidad de los resultados. Se evaluaron y documentaron los siguientes parámetros: el tipo de establecimiento (público o privado); la población cubierta; el número de pacientes y exámenes; los equipos radiológicos, los de procesamiento de imágenes y los suministros; la educación y la capacitación del personal profesional y técnico; los programas de la garantía de la calidad y del mantenimiento preventivo, y la adherencia a las normas de seguridad radiológica. Se determinaron el funcionamiento de los equipos de rayos $X$, los receptores de la imagen y las procesadoras; las condiciones del cuarto oscuro y de la visualización de las imágenes; las dosis recibidas por los pacientes y la calidad de la imagen, usando parámetros uniformados en todos los casos. Los paneles independientes de radiólogos, reconocidos como expertos por la sociedad radiológica local, evaluaron la calidad de las imágenes clínicas obtenidas y realizaron una interpretación radiológica para cada paciente usando las mismas películas e historia clinica a disposición de los médicos especialistas en imaginología de la institución. El acuerdo entre los informes de los paneles de expertos y los de los radiólogos locales se tomó como un indicador de la certeza de la interpretación radiológica.

Resultados. Se analizaron 366 mamografías, 343 procedimientos radiológicos para las quejas del aparato digestivo, 319 exámenes de rayos X de la columna vertebral y 157 radiografías de tórax. El acuerdo entre la interpretación radiológica del panel de expertos y del médico local fue de $70 \%$ a $100 \%$, excepto en el caso de las películas de la columna vertebral en Cuba $(57,8 \%)$ y de las mamografías en México (33,3\%), que el panel de expertos juzgó estaban entre las imágenes clínicas de peor calidad. Se encontró una correlación positiva significativa entre la certeza en la interpretación radiológica y la calidad de las imágenes radiológicas. La calidad de la imagen mostró una correlación positiva con el nivel de formación y capacitación de los técnicos. Los estudios que se realizaron en los servicios que contaban con equipos automáticos de revelado y que cumplieron con los indicadores establecidos para el contacto película-pantalla obtuvieron imágenes de mejor calidad y una proporción mayor de estudios con resultados concordantes. Más de $50 \%$ de los negatoscopios no cumplieron con los criterios de calidad para el brillo y la homogeneidad. 
Conclusiones. Una imagen de buena calidad es fundamental para el logro de un diagnóstico certero. Se debe hacer hincapié en la educación continuada de los técnicos de radiología y en la adquisición y el mantenimiento de los equipos y los accesorios adecuados, especialmente de los negatoscopios, las pantallas intensificadoras y las máquinas reveladoras automáticas, dada la repercusión que tienen en la calidad de las imágenes.

En América Latina y el Caribe, como en el resto del mundo, los estudios de radiodiagnóstico constituyen una de las técnicas más utilizadas para confirmar el diagnóstico de algunas de las enfermedades más frecuentes - como la tuberculosis y las fracturas óseas-o potencialmente mortales - como las enfermedades cardiovasculares y neoplásicas-, cuya incidencia va en aumento en la Región (1-3). Prácticamente todas las especialidades médicas utilizan el diagnóstico radiológico, lo que ha llevado a que varias asociaciones científicas elaboren recomendaciones para regular la prescripción de los exámenes de diagnóstico radiológico, los exámenes más adecuados para cada enfermedad y los criterios anatómicos que deben emplearse para evaluar la calidad de las imágenes (4), entre otros aspectos.

Debido a que la complejidad tecnológica ha llevado al incremento de las dosis de radiación recibidas por los pacientes, se ha dedicado un gran esfuerzo a analizar los posibles efectos perjudiciales de la irradiación con fines médicos. Las dosis aplicadas a los pacientes sometidos a exámenes radiológicos se comenzaron a medir en los Estados Unidos de América en la década de 1950 (5). En la década siguiente, los gobiernos de los Estados Unidos, el Reino Unido y Nueva Zelanda, entre otros, documentaron las exposiciones de poblaciones a los rayos $\mathrm{X}$ para procedimientos médicos (6). Más tarde, en esa misma década, se desarrollaron programas de control de la calidad y normas radiológicas clínicas que recibieron el apoyo de las sociedades profesionales y de organizaciones no gubernamentales con competencia en el tema. Desde entonces, se han publicado cientos de documentos, principalmente sobre las mediciones de niveles de referencia en radiodiagnóstico, propuestos inicialmente por la Junta Nacional de Protección Radiológica (National Radiological Protection Board, NRPB) del Reino Unido y luego incorporados como "niveles orientativos" en las Normas básicas internacionales de seguridad para la protección contra las radiaciones ionizantes y para la seguridad de las fuentes de radiación (BSS) (7).

La Directiva sobre Exposiciones Médicas 97/43 (8) les exige a los países de la Unión Europea que controlen las exposiciones de los pacientes mediante la asignación de responsabilidades, la medición de las dosis de radiación y la documentación de programas de garantía de la calidad. Otros go- biernos han adoptado o están en proceso de adoptar o adaptar las BSS y se han publicado varios trabajos sobre mediciones físicas (9-14). Sin embargo, se ha hecho muy poco por evaluar integralmente la calidad de los servicios junto con los aspectos organizativos y dosimétricos.

En 1999, el Programa de Radiología y Radioprotección de la Organización Panamericana de la Salud (OPS) convocó un concurso para la presentación de proyectos de investigación con el tema "Evaluación de la calidad de los servicios de radiodiagnóstico". Cinco países -Argentina, Bolivia, Colombia, Cuba y México- recibieron contratos de investigación. Aunque las instalaciones de radiodiagnóstico de estos países se encontraban en diferentes niveles de desarrollo técnico y socioeconómico, los proyectos de investigación presentados tenían tantos aspectos comunes, que la OPS decidió — respetando las particularidades de cada proyecto- reunirlos en un "estudio multicéntrico" y asignarle la coordinación al investigador principal de Argentina. El objetivo central de esa investigación fue determinar la correlación entre algunos indicadores de calidad de los servicios de imaginología con la certeza en la interpretación radiológica de cuatro afecciones frecuentes en los cinco países participantes. Esta publicación describe los resultados de ese estudio multicéntrico.

\section{MATERIALES Y MÉTODOS}

Se evaluó la calidad de los servicios de radiología en el diagnóstico de cuatro afecciones (masas en los senos, trastornos gastrointestinales, dolor de espalda y tuberculosis) en el período comprendido entre septiembre de 2000 y diciembre de 2001. En el estudio participaron 26 servicios de radiología: 5 de Argentina (Rosario), 7 de Bolivia (La Paz, Cochabamba, Santa Cruz y Sucre), 4 de Colombia (Santa Fe de Bogotá y Cali), 5 de Cuba (Ciudad de La Habana, Santa Clara y Santiago de Cuba) y 5 de México (México, D.F.). Todos los servicios, tanto públicos como privados, tenían una capacidad tecnológica intermedia, considerada del grado de complejidad tecnológica II, según la clasificación propuesta por la OPS (15).

Los investigadores participantes seleccionaron por consenso las enfermedades, los exámenes y los servicios que se evaluarían, teniendo en cuenta las afecciones más frecuentes y los exámenes que requieren altos niveles de exposición a la radiación y un mayor número de proyecciones radiográficas. Como indicadores de calidad de los servicios se emplearon: la calidad de las imágenes (identificación y etiquetado, posicionamiento del paciente, artefactos y contraste y latitud); el estado de los equipos de 
CUADRO 1. Afecciones estudiadas, según el país y el tipo de institución (pública o privada)

\begin{tabular}{|c|c|c|c|c|c|c|c|c|c|c|c|}
\hline \multirow{2}{*}{$\begin{array}{l}\text { Diagnóstico } \\
\text { o síntoma }\end{array}$} & \multirow[b]{2}{*}{ Procedimiento } & \multirow[b]{2}{*}{ Equipo } & \multicolumn{2}{|c|}{ Argentina } & \multicolumn{2}{|c|}{ Bolivia } & \multicolumn{2}{|c|}{ Colombia } & \multirow{2}{*}{$\frac{\text { Cuba }}{\text { Pública }}$} & \multicolumn{2}{|c|}{ México } \\
\hline & & & Pública & Privada & Pública & Privada & Pública & Privada & & Pública & Privada \\
\hline Masas en & Tamizaje & Mamógrafo & 2 & 3 & $N D^{a}$ & ND & 2 & 2 & ND & ND & 3 \\
\hline$(n=366)$ & $\begin{array}{l}\text { Mamografía } \\
\text { diagnóstica }\end{array}$ & Mamógrafo & 2 & 3 & 2 & 1 & 2 & 2 & 2 & 2 & 3 \\
\hline $\begin{array}{l}\text { Trastornos } \\
\text { gastro- }\end{array}$ & Enema de colon & $\begin{array}{l}\text { Fluoroscopio } \\
\text { y seriógrafo }\end{array}$ & 2 & 2 & ND & ND & 2 & 2 & 4 & ND & ND \\
\hline$(n=343)$ & $\begin{array}{l}\text { Radiografía } \\
\text { seriada de } \\
\text { esófago- } \\
\text { estómago-duodeno }\end{array}$ & $\begin{array}{l}\text { Fluoroscopio } \\
\text { y seriógrafo }\end{array}$ & 2 & 2 & ND & ND & 2 & 2 & 4 & ND & ND \\
\hline $\begin{array}{l}\text { Dolor en } \\
\text { la espalda }\end{array}$ & $\begin{array}{l}\text { Radiografía de } \\
\text { columna } \\
\text { lumbosacra }\end{array}$ & $\begin{array}{l}\text { Rayos X y } \\
\text { tomógrafo } \\
\text { computarizado }\end{array}$ & 1 & 3 & 3 & 1 & 2 & 2 & 4 & ND & ND \\
\hline$(n=319)$ & $\begin{array}{l}\text { Radiografía } \\
\text { de columna } \\
\text { cervical }\end{array}$ & $\begin{array}{l}\text { Rayos } X \text { y } \\
\text { tomógrafo } \\
\text { computarizado }\end{array}$ & 1 & 3 & 3 & 1 & 2 & 2 & 4 & ND & ND \\
\hline $\begin{array}{l}\text { Tuberculosis } \\
(n=157)\end{array}$ & $\begin{array}{l}\text { Radiografía } \\
\text { de tórax }\end{array}$ & Rayos X & ND & ND & 3 & 1 & 2 & 2 & ND & ND & ND \\
\hline
\end{tabular}

${ }^{a}$ ND: No hay datos.

rayos $X$ (evaluación mecánica, coincidencia de los campos luminosos y de radiación, alineación, exactitud y reproducibilidad de la tensión, exactitud y reproducibilidad del tiempo, linealidad del producto corriente-tiempo, espesor hemirreductor); las condiciones de revelado y visualización (tipo de revelado, contacto película-pantalla, luminosidad y uniformidad del brillo de los negatoscopios); y las características del personal (titulación, años de experiencia y cursos de formación continuada y estudios de protección radiológica realizados).

\section{La selección de los pacientes}

Cada país debía reclutar un mínimo de 20 pacientes por cada tipo de estudio radiológico y por cada servicio participante. La selección de los pacientes se efectuó entre julio de 2000 y febrero de 2001, según el siguiente calendario: estudios de mama (julio-septiembre de 2000), de columna vertebral (octubre-noviembre de 2000), de trastornos gastrointestinales (diciembre de 2000-febrero de 2001) y de tuberculosis (enero-febrero de 2001). Se aceptaron todos los pacientes adultos de 18 a 70 años de edad, de uno u otro sexo, que acudieron a los servicios de radiología con una prescripción para realizarse un examen diagnóstico por alguna de las afecciones abarcadas por el estudio (cuadro 1). Se excluyeron los casos de urgencia. Se obtuvo el con- sentimiento informado de todos los pacientes y se garantizó la confidencialidad de los resultados.

En cada país, la sociedad nacional de radiología o el organismo colegiado correspondiente conformaron un panel evaluador de expertos para cada tipo de examen radiológico a propuesta del investigador principal. Estos paneles estaban integrados por un número impar de expertos en radiología, siempre más de tres. Los expertos eran médicos especialistas en radiología o mastología con al menos 5 años de experiencia en la especialidad y probada experiencia en el diagnóstico de enfermedades vinculadas con los estudios radiológicos evaluados. En algunos casos, los paneles participaron en la evaluación de más de un tipo de estudio si contaban con los conocimientos clínicos requeridos. En total en este estudio participaron 182 técnicos y profesionales (cuadro 2).

\section{Documentación}

Cada institución describió el personal participante (radiólogos, técnicos de rayos X, enfermeros, técnicos de mantenimiento, físicos médicos y oficiales de radioprotección) y su calificación (especialización certificada, años de experiencia total y en la técnica en particular y actividades de actualización profesional realizadas en los últimos 5 años).

La protección radiológica se evaluó mediante una inspección visual especializada que valoró el 
CUADRO 2. Personal que participó en la investigación, según el país

\begin{tabular}{lrrrrrr}
\hline \multicolumn{1}{c}{ Personal } & Argentina & Bolivia & Colombia & Cuba & México & Total \\
\hline Investigadores & 5 & 4 & 7 & 11 & 11 & 38 \\
Médicos institucionales & 14 & 11 & 12 & 30 & 7 & 74 \\
Expertos de los paneles & 15 & 5 & 16 & 10 & 3 & 49 \\
Otros & 3 & 0 & 5 & 10 & 3 & 21 \\
$\quad$ Total & 37 & 20 & 40 & 61 & 24 & 182 \\
\hline
\end{tabular}

blindaje estructural, las medidas y medios de protección y el sistema de dosimetría personal. Se verificó si el servicio contaba con la habilitación de la autoridad reguladora nacional, si las técnicas radiológicas para cada equipo (tensión $[\mathrm{kVp}]$, producto corriente-tiempo [mAs], distancia foco-película, etc.) estaban descritas en una carta técnica y se encontraban en un lugar visible y si se contaba con un manual de procedimientos. Además, se solicitó una descripción de los programas de mantenimiento preventivo y correctivo y del programa de garantía de la calidad (responsable, tipo de evaluación, frecuencia y si abarcaba el cuarto oscuro, los sistemas de recepción de la imagen y el equipo de rayos X). Se verificó si se contaba con las estadísticas de todos estos procedimientos.

En el cuaderno de recolección de la información de los pacientes se anotaron los datos de la prescripción del examen radiológico, la fecha de realización, la técnica radiológica empleada, las imágenes diagnósticas obtenidas y el informe radiológico completo, así como el nombre del técnico que ejecutó el examen y el del radiólogo local que emitió el informe. Siempre que fue posible se recogieron también los resultados de las comprobaciones clínicas efectuadas después del examen radiológico (endoscopias, cirugías, análisis clínicos de laboratorio, estudios de anatomía patológica, entre otros). Estos resultados fueron posteriormente comparados con los informes radiológicos emitidos por el radiólogo local y por el panel de expertos.

Para garantizar la confidencialidad del paciente, del radiólogo y de la institución, el investigador responsable guardó el informe del radiólogo local y entregó al panel de expertos las radiografías (después de ocultar la identificación con etiquetas removibles), acompañadas de una copia anónima de la prescripción del examen y de la información clínica pertinente. Las radiografías se presentaron al panel evaluador de manera aleatoria, identificadas mediante códigos que se mantuvieron en estricta confidencia. Al terminar la evaluación del panel, las radiografías se devolvieron a la institución de procedencia en sus sobres originales.

\section{Evaluación técnica}

Antes de realizar los exámenes radiológicos se hizo una evaluación del equipo instalado en cada servicio. El comportamiento técnico de las unidades de rayos $\mathrm{X}$, los receptores de la imagen, las máquinas reveladoras, así como las condiciones del cuarto oscuro y de la sala de lectura se evaluaron según los criterios del Consejo Nacional de Radioprotección y Medidas de Radiación (National Council on Radiation Protection and Measurements, NCRP) de los Estados Unidos de América (16), mientras que para los equipos de mamografía se emplearon los del Colegio Estadounidense de Radiología (American College of Radiology, ACR) (17). Además, se realizó una evaluación física de la calidad de las imágenes obtenidas en los mamógrafos mediante un patrón de resolución y el análisis de una mamografía efectuada a un maniquí del ACR. Este maniquí simulaba una mama de $4,2 \mathrm{~cm}$ de espesor, compuesta por $50 \%$ de tejido adiposo y $50 \%$ de tejido glandular, con elementos interiores que simulaban diferentes enfermedades de la mama. La calidad de la imagen se evaluó según el número de enfermedades que se podían visualizar en la radiografía tomada al maniquí y los pares de líneas que se podían observar claramente en la imagen del patrón de resolución.

La dosis de radiación recibida en la superficie de los pacientes en los estudios de columna vertebral, vías digestivas y tórax y la dosis glandular promedio en los casos de mamografías se calcularon a partir del kerma en el aire, medido con un electrómetro y una cámara de ionización calibrados por un laboratorio de dosimetría acreditado. Para realizar el cálculo de la dosis se utilizaron los parámetros empleados habitualmente para cada examen radiológico (tensión, producto corriente-tiempo, distancia foco-película, etc.). Los valores de dosis resultantes se compararon con los niveles de dosis orientativos de las BSS (7).

La certeza en la interpretación radiológica fue evaluada por el equipo responsable de la investigación en cada país. Para ello se tuvo en cuenta la coincidencia del informe emitido por el radiólogo local con el emitido por el panel de expertos. Este realizó el 
informe de las imágenes radiográficas después que lo hiciera el radiólogo local y se garantizó que ambos tuvieran acceso a la misma información. El panel diagnosticó por mayoría las enfermedades primaria y secundaria y, según la técnica empleada, evaluó la calidad general de la imagen de la radiografía (posición del paciente, llenado con el líquido de contraste, cantidad de proyecciones, y contraste y latitud de la placa radiográfica), la forma de identificación, el etiquetado de la radiografía y la presencia de artefactos. La evaluación se realizó según una escala de 1 (no útiles) a 5 (de calidad óptima). Se consideró como diagnóstico correcto el realizado por el panel de expertos. Las radiografías evaluadas por el panel como "no útiles" se consideraron también como no coincidentes (diagnóstico incorrecto).

\section{Correlaciones estadísticas}

Para el análisis estadístico se utilizó el programa estadístico SPSS, versión 10. Se estableció un nivel de significación de 0,05 y se calcularon los intervalos de confianza de 95\% (IC95\%). Se realizaron diagramas de dispersión, ecuaciones de regresión y análisis de la varianza entre las variables cuantificadas para determinar cuál de los indicadores de calidad se correlacionaba mejor con la coincidencia entre en el diagnóstico del radiólogo institucional y el del panel de expertos por una parte, y la calidad de la imagen de las películas clínicas evaluadas por el panel de expertos, por la otra.

\section{RESULTADOS}

\section{Documentación}

Todos los médicos que participaron en el estudio tenían más de 5 años de experiencia en su especialidad, excepto dos que realizaban estudios de mamografía en Argentina y México y tenían 1 y 2 años de experiencia, respectivamente. Todos los médicos habían recibido educación continuada, excepto en Bolivia (cuadro 3), donde los únicos con formación especializada fueron los que intervinieron en los estudios de mamografía; sin embargo, ninguno de estos últimos había recibido capacitación en protección radiológica.

Todos los técnicos participantes (llamados tecnólogos en algunos países) habían recibido entrenamiento formal (cuadro 4). Sin embargo, solo en los servicios de mamografía de Argentina, Colombia y Cuba se encontraron técnicos con formación universitaria. Todos los técnicos habían recibido algún curso de educación continuada, excepto en Bolivia, donde solo poco más de la mitad de los técnicos participantes había asistido a alguno de esos cursos.

En los centros hospitalarios participantes bolivianos que aportaron casos de tuberculosis y co-

CUADRO 3. Características de los médicos imaginólogos en los servicios que participaron en la investigación, según la especialidad y el país

\begin{tabular}{|c|c|c|c|c|c|c|}
\hline \multirow[b]{2}{*}{ Especialidad y país } & \multirow[b]{2}{*}{ No. } & \multicolumn{2}{|c|}{ Años de experiencia } & \multirow[b]{2}{*}{ Especialidad } & \multirow{2}{*}{$\begin{array}{l}\text { Personal } \\
\text { capacitado } \\
(\%)\end{array}$} & \multirow{2}{*}{$\begin{array}{l}\text { Capacitación } \\
\text { en protección } \\
\text { radiológica, horas }\end{array}$} \\
\hline & & Mínimo & Máximo & & & \\
\hline \multicolumn{7}{|l|}{ Columna vertebral } \\
\hline Argentina & 9 & 5 & $\mathrm{ND}^{\mathrm{b}}$ & Radiólogo & 100 & 25 \\
\hline Bolivia & 10 & 7 & $>10$ & Radiólogo & 20,0 & 25 \\
\hline Colombia & 18 & 7 & $>10$ & Radiólogo & 100 & 20 \\
\hline Cuba & 25 & 12 & 25 & Radiólogo & 100 & 40 \\
\hline \multicolumn{7}{|l|}{ Tuberculosis } \\
\hline Bolivia & 13 & 7 & $>10$ & Radiólogo & 15,3 & 25 \\
\hline Colombia & 18 & 7 & $>10$ & Radiólogo & 100 & 20 \\
\hline \multicolumn{7}{|c|}{ Trastornos gastrointestinales } \\
\hline Argentina & 12 & 10 & $>10$ & Radiólogo & 100 & 25 \\
\hline Colombia & 9 & 7 & $>10$ & Radiólogo & 100 & 20 \\
\hline Cuba & 25 & 12 & 25 & Radiólogo & 100 & 40 \\
\hline \multicolumn{7}{|l|}{ Mama } \\
\hline Argentina & 8 & 2 & 10 & $\begin{array}{l}3 \text { radiólogos } \\
5 \text { mastólogos }\end{array}$ & 100 & 25 \\
\hline Bolivia & 5 & 10 & $>10$ & $\begin{array}{l}3 \text { radiólogos } \\
2 \text { mastólogos }\end{array}$ & 100 & 0 \\
\hline Colombia & 6 & 6 & $>10$ & Radiólogo & 100 & 20 \\
\hline Cuba & 3 & 6 & $>10$ & Radiólogo & 100 & 112 \\
\hline México & 9 & 1 & $>10$ & Radiólogo & 100 & 20 \\
\hline
\end{tabular}

a Promedio de horas de estudio acumuladas per cápita en cualquier momento.

${ }^{b}$ ND: No hay datos. 
CUADRO 4. Características de los técnicos que participaron en la investigación, según la especialidad y el país

\begin{tabular}{|c|c|c|c|c|c|c|}
\hline \multirow[b]{2}{*}{ Especialidad y país } & \multirow[b]{2}{*}{ No. } & \multicolumn{2}{|c|}{ Años de experiencia } & \multirow{2}{*}{$\begin{array}{l}\text { Nivel en la } \\
\text { especialidad }\end{array}$} & \multirow{2}{*}{$\begin{array}{l}\text { Personal } \\
\text { capacitado } \\
\text { (\%) }\end{array}$} & \multirow{2}{*}{$\begin{array}{l}\text { Capacitación } \\
\text { en protección } \\
\text { radiológica, horas }\end{array}$} \\
\hline & & Mínimo & Máximo & & & \\
\hline \multicolumn{7}{|l|}{ Columna vertebral } \\
\hline Argentina & $N D^{b}$ & ND & ND & ND & ND & ND \\
\hline Bolivia & 15 & 2 & $>10$ & Técnico & 53,3 & 25 \\
\hline Colombia & 73 & 5 & $>12$ & Técnico & 100 & 40 \\
\hline Cuba & 45 & 11 & $>20$ & Técnico & 100 & 40 \\
\hline \multicolumn{7}{|l|}{ Tuberculosis } \\
\hline Bolivia & 25 & 10 & ND & Técnico & 56,0 & 25 \\
\hline Colombia & 73 & 5 & $>12$ & Técnico & 100 & 40 \\
\hline \multicolumn{7}{|c|}{ Trastornos gastrointestinales } \\
\hline Argentina & ND & ND & ND & ND & ND & ND \\
\hline Colombia & 10 & 5 & $>12$ & Técnico & 100 & 40 \\
\hline Cuba & 45 & 11 & $>20$ & Técnico & 100 & 40 \\
\hline \multicolumn{7}{|l|}{ Mama } \\
\hline Argentina & 6 & 3 & $>10$ & Universitario & 100 & 15 \\
\hline Bolivia & 4 & 3 & 10 & Técnico & 25,0 & 25 \\
\hline Colombia & 9 & ND & ND & Universitario & 100 & 40 \\
\hline Cuba & 5 & 6 & $>10$ & Universitario & 100 & 112 \\
\hline México & 12 & 3 & $>10$ & Técnico & 100 & 20 \\
\hline
\end{tabular}

a Promedio de horas de estudio acumuladas per cápita en cualquier momento.

${ }^{b} \mathrm{ND}$ : No hay datos.

CUADRO 5. Disponibilidad de otros profesionales de la salud en los servicios que participaron en la investigación, según la especialidad y el país

\begin{tabular}{|c|c|c|c|}
\hline Especialidad y país & Físicos médicos ${ }^{a}$ & $\begin{array}{l}\text { Ingenieros de } \\
\text { mantenimiento }\end{array}$ & $\begin{array}{l}\text { Hay un oficial de } \\
\text { protección radiológica } \\
\text { en la institución }\end{array}$ \\
\hline \multicolumn{4}{|l|}{ Columna vertebral } \\
\hline Argentina & $\mathrm{ND}^{\mathrm{b}}$ & 1 & Sí \\
\hline Bolivia & 0 & 0 & Sí \\
\hline Colombia & 1 & 0 & No \\
\hline Cuba & 1 & 1 & Sí \\
\hline \multicolumn{4}{|l|}{ Tuberculosis } \\
\hline Bolivia & 0 & 0 & Sí \\
\hline Colombia & 0 & 0 & No \\
\hline \multicolumn{4}{|c|}{ Trastornos gastrointestinales } \\
\hline Argentina & 0 & 1 & Sí \\
\hline Colombia & 1 & 1 & No \\
\hline Cuba & 1 & 1 & Sí \\
\hline \multicolumn{4}{|l|}{ Mama } \\
\hline Argentina & $2+1^{c}$ & 2 & Sí \\
\hline Bolivia & 1 & 1 & Sí \\
\hline Colombia & 1 & 1 & No \\
\hline Cuba & 1 & 2 & Sí \\
\hline México & 1 & 2 & Sí \\
\hline
\end{tabular}

lumna vertebral y en los colombianos con casos de tuberculosis no se contaba con físicos médicos ni con ingenieros de mantenimiento (cuadro 5). Tampoco había físicos médicos en los centros argentinos que participaron en los estudios por trastornos gastrointestinales. En Colombia, ninguno de los cen- tros participantes en esta investigación contaba con un oficial de radioprotección.

Todos los países informaron contar con órganos reguladores y normas nacionales para la seguridad radiológica. Sin embargo, solo en cuatro centros (dos de Bolivia y dos de Colombia) se en- 
contraron manuales de protección radiológica. Ninguno de los centros participantes de esos dos países contaba con programas habituales para la medición de las dosis de radiación que recibían los pacientes; solo uno de los centros de México tenía un programa de este tipo. No se informó de que hubiera algún programa de análisis del rechazo de películas en ninguno de los centros de Cuba.

Solo dos de los servicios colombianos participantes habían instaurado programas periódicos de control de la calidad para todos los equipos. Bolivia y México mostraron los mejores indicadores en cuanto al mantenimiento de los equipos y en todas sus instituciones había programas que abarcaban tanto el mantenimiento correctivo como el preventivo.

\section{Evaluación técnica}

Seguridad radiológica. En todos los países que participaron en la investigación, las normas nacionales habían adoptado los límites de dosis recomendados por las BSS para los trabajadores (7). La dosimetría del personal se llevaba a cabo mediante dosímetros termoluminiscentes con una frecuencia de recambio mensual, excepto en dos hospitales de Cuba donde se utilizaban dosímetros de película que se cambiaban cada dos meses. El análisis de los resultados de la dosimetría personal en cada servicio demostró que en ningún caso se sobrepasaron los límites de dosis para los trabajadores.

Evaluación de los equipos de rayos $X$. Según la evaluación realizada en cada servicio, más de $30 \%$ de las unidades de radiología convencional y fluoroscopia presentaba fallas, fundamentalmente en elementos mecánicos y geométricos. En las unidades de mamografía, la colimación del haz de radiación era inadecuada en $30 \%$ de los equipos. Además, el control automático de la exposición no logró compensar el nivel de radiación según el espesor de la mama en $25 \%$ de los equipos evaluados, lo que incidió negativamente en la densidad óptica de las imágenes.

Revelado y visualización. En los servicios de mamografía, donde la complejidad del tejido a estudiar y de las posibles alteraciones obligan a observar estándares muy elevados de calidad de la imagen y de su visualización, la mayoría de los negatoscopios se encontraban muy por debajo de la norma de 3000 candelas por metro cuadrado, establecida por el ACR (17). Solamente un negatoscopio (en una unidad de Bolivia) tenía la iluminación, la homogeneidad y el color adecuados. En cuanto al proceso de revelado, solo 3 de las 20 reveladoras evaluadas se sometían a pruebas diarias de sensito- metría y densitometría y solo 10 reveladoras eran del uso exclusivo de los servicios de mamografía, un requisito de calidad indispensable en este tipo de estudio. En total se analizaron 16 reveladoras y cuartos oscuros dedicados al resto de las enfermedades estudiadas en igual número de departamentos de radiología. En solo cinco de ellos se realizaba el control diario de las condiciones de revelado y solo en la mitad de esos servicios se habían instaurado programas de análisis del porcentaje de películas rechazadas. Los negatoscopios fueron los componentes que más fallas mostraron en todos los estudios, ya que más de $50 \%$ de ellos no cumplieron con los criterios de calidad en cuanto a brillo y homogeneidad. También se encontraron fallas en cerca de $25 \%$ de los cuartos oscuros, fundamentalmente por la entrada de luz del exterior y la intensidad excesiva de las luces de seguridad.

La dosis administradas a los pacientes. Las dosis de radiación, tanto la de entrada en la superficie del paciente como la dosis glandular promedio en el caso de las mamografías, se calcularon a partir de las lecturas realizadas con cámaras de ionización, excepto en Bolivia, donde se midieron de forma directa mediante dosímetros termoluminiscentes. En Cuba se calcularon además las dosis efectivas y las recibidas por los pacientes en los órganos. Todas las dosis - calculadas o medidas- se mantuvieron por debajo de los niveles propuestos por las BSS (7), con excepción de los estudios de tórax realizados en Bolivia, donde se utilizaron técnicas de muy baja tensión, y los estudios de columna vertebral realizados en Cuba, donde por lo general se utilizaron valores del producto corriente-tiempo mayores que los habituales.

\section{La calidad de la imagen}

En total se analizaron 366 mamografías, 343 exámenes por trastornos gastrointestinales, 319 de columna vertebral y 157 de tórax. La evaluación de la calidad de las imágenes radiográficas se basó en general en el criterio de los paneles de expertos (cuadro 6). El análisis comparativo de los informes radiológicos emitidos por el panel de expertos y por los radiólogos locales en los tres países que estudiaron casos de trastornos gastrointestinales demostró que la calidad de la imagen fue buena en menos de $70 \%$ de los estudios (69,4\% en Argentina, 62,5\% en Colombia y $64,5 \%$ en Cuba). A pesar de que en Cuba todos los estudios se realizaron con equipos de radiografía convencional y en Argentina y Colombia se emplearon equipos de fluoroscopia, las calificaciones fueron muy similares en los tres países. Sin embargo, esta situación parece haber inci- 
CUADRO 6. Resultados de la evaluación de las placas radiográficas por el panel de expertos y su coincidencia en la interpretación radiológica con los radiólogos locales

\begin{tabular}{|c|c|c|c|c|c|c|c|}
\hline \multirow[b]{2}{*}{ Especialidad y país } & \multirow[b]{2}{*}{$\begin{array}{c}\text { Total de } \\
\text { estudios } \\
\text { analizados }\end{array}$} & \multicolumn{4}{|c|}{ Estudios con calidad óptima, \% } & \multicolumn{2}{|c|}{ Coincidencia } \\
\hline & & $\begin{array}{l}\text { Identificación } \\
\text { y etiquetado }\end{array}$ & $\begin{array}{c}\text { Posicionamiento } \\
\text { del } \\
\text { paciente }\end{array}$ & Artefactos & $\begin{array}{l}\text { Calidad } \\
\text { de la } \\
\text { imagen }\end{array}$ & No. & $\%$ \\
\hline \multicolumn{8}{|l|}{ Columna vertebral } \\
\hline Argentina & 57 & $N D^{a}$ & 72,0 & 73,7 & 77,2 & 46 & 80,7 \\
\hline Bolivia & 50 & 65,0 & 69,2 & ND & 48,6 & 50 & 100,0 \\
\hline Colombia & 79 & 79,7 & 64,5 & 69,6 & 41,8 & 64 & 81,0 \\
\hline Cuba & 237 & 70,4 & 49,8 & 37,1 & 37,1 & 137 & 57,8 \\
\hline \multicolumn{8}{|l|}{ Tuberculosis } \\
\hline Bolivia & 78 & ND & ND & ND & 41,0 & 78 & 100,0 \\
\hline Colombia & 79 & 67,0 & 82,3 & 78,5 & 81,0 & 72 & 91,1 \\
\hline \multicolumn{8}{|l|}{$\begin{array}{l}\text { Trastornos } \\
\text { gastrointestinales }\end{array}$} \\
\hline Argentina & 62 & ND & 82,2 & 100,0 & 69,4 & 44 & 71,0 \\
\hline Colombia & 56 & 75,0 & 92,8 & 76,8 & 62,5 & 46 & 82,1 \\
\hline Cuba & 237 & 98,3 & 64,5 & 69,2 & 64,5 & 173 & 73,0 \\
\hline \multicolumn{8}{|l|}{ Mama } \\
\hline Argentina & 96 & 100,0 & 78,1 & 85,4 & 71,8 & 95 & 98,9 \\
\hline Bolivia & 37 & 50,0 & 41,8 & ND & 35,9 & 37 & 100,0 \\
\hline Colombia & 69 & 17,4 & 68,1 & 75,3 & 85,5 & 52 & 75,4 \\
\hline Cuba & 80 & 97,5 & 73,8 & 81,2 & 80,0 & 67 & 83,8 \\
\hline México & 87 & 64,3 & 16,1 & 0,46 & 0,46 & 29 & 33,3 \\
\hline
\end{tabular}

a ND: No hay datos.

dido en los bajos resultados obtenidos en Cuba en cuanto al posicionamiento correcto del paciente (solo $64,5 \%$ de los casos tuvieron una adecuada posición) en comparación con los otros dos países. Excepto en Argentina (77,2\%), menos de 50\% de los estudios de columna vertebral tuvieron una buena calidad de la imagen, fundamentalmente por errores de posicionamiento y la presencia de artefactos.

En los servicios de mamografía, los aspectos que más incidieron en la mala calidad de las imágenes, según el criterio del panel de expertos, fueron el mal posicionamiento del paciente y la identificación y el etiquetado deficientes de las películas. A pesar de que en la evaluación de la calidad de la imagen realizada con el maniquí del ACR, cerca de $80 \%$ de los sistemas tuvieron resultados satisfactorios, se encontró una notable heterogeneidad en la calidad de las imágenes obtenidas en los diferentes países participantes. En los servicios de mamografía, el porcentaje de imágenes con calidad óptima fue muy bajo en México (0,46\%), mientras que en Colombia $(85,5 \%)$ este parámetro tuvo el mejor desempeño (cuadro 6).

La coincidencia en la interpretación radiológica entre el panel de expertos y el radiólogo local de cada servicio se mantuvo por encima de $70 \%$ en todos los casos, excepto en los estudios de columna vertebral en Cuba $(57,8 \%)$ y los de mama en México $(33,3 \%)$, que según el panel de expertos produjeron las imágenes clínicas de peor calidad. Cuando los diagnósti- cos no coincidieron, se informó a las instituciones de esta discrepancia y en algunos casos se requirió la intervención de otros expertos para garantizar el tratamiento clínico adecuado de los pacientes.

En los estudios de mama en Cuba, donde el panel de expertos revisó 255 películas y coincidió con la interpretación de los radiólogos institucionales en 68 de los 80 pacientes, se realizaron biopsias por aspiración con aguja fina a 27 pacientes, como parte de la conducta diagnóstico-terapéutica programada. En 21 de ellos, la biopsia confirmó el diagnóstico coincidente del panel y el médico institucional. En los otros seis casos, tres biopsias confirmaron el informe radiológico del centro y tres el del panel de expertos. Este resultado podría explicarse por el hecho de que el radiólogo local determina sus propios estándares de calidad en las imágenes (por ejemplo, la densidad óptica de fondo), mientras que el panel de expertos debió evaluar imágenes provenientes de diferentes servicios con una variedad mucho mayor de estándares de calidad de la imagen.

\section{La certeza en la interpretación radiológica}

En todos los tipos de estudios se encontró una relación directa significativa entre la certeza en la interpretación radiológica y la calidad de las imágenes radiográficas $(P \leq 0,002)$. En todos los casos, 
los diagnósticos coincidentes correspondieron a los exámenes con una buena calidad de la imagen, según el criterio del panel.

En los servicios de mamografía, los resultados de las mediciones físicas de la calidad de la imagen con patrones de resolución se correspondieron con los obtenidos con el maniquí del ACR $(P<0,001)$. No se encontró relación alguna entre el resultado de la evaluación con el maniquí del ACR y la dosis glandular promedio $(P=0,2)$.

Se encontró una relación significativa entre la coincidencia en la interpretación radiológica del panel de expertos y el radiólogo local y los años de experiencia de este último en los estudios de mamografía $(P=0,015)$ y de columna vertebral $(P=0,03)$.

La calidad de la imagen fue directamente proporcional al nivel de formación y capacitación de los técnicos $(P<0,024)$. Además, se encontró una asociación inversa entre los años de experiencia de los técnicos y la calidad de las imágenes radiográficas en los estudios por trastornos gastrointestinales $(P<0,001)$ y de columna vertebral $(P=0,001)$. Esta relación inversa pudiera explicarse por el hecho de que los técnicos más jóvenes reciben más capacitación continuada por manifestar más interés o por los procedimientos internos que se siguen en estos departamentos. Por ejemplo, en uno de los departamentos en Cuba se realizaba habitualmente una evaluación preliminar de la calidad de las radiografías inmediatamente después del proceso de revelado y antes de enviarlas al radiólogo informante, pero estas evaluaciones se centraban en los exámenes realizados por los técnicos más jóvenes e inexpertos. Este tipo de procedimientos puede haber influido en este resultado.

La evaluación de los equipos instalados en cada servicio reveló que la certeza en la interpretación radiológica estaba asociada con el tipo de revelado $(P<0,019)$ y con el estado de la combinación película-pantalla $(P<0,001)$. Los estudios realizados en los servicios que contaban con equipos automáticos de revelado y que cumplieron con los indicadores relacionados con el contacto películapantalla obtuvieron imágenes de mejor calidad y una mayor proporción de estudios coincidentes, mientras que los peores resultados en cuanto a la calidad de la imagen se obtuvieron en los servicios donde se efectuaba el revelado manual.

\section{DISCUSIÓN}

La evaluación de los departamentos de radiología diagnóstica es de gran importancia debido al impacto que pueden tener las interpretaciones radiológicas acertadas en el tratamiento de los pa- cientes, los costos de funcionamiento de estos servicios y los posibles efectos dañinos de las radiaciones ionizantes.

Aunque los estudios dosimétricos abundan, esta es la primera investigación que intenta relacionar la exactitud en la interpretación radiológica con los aspectos organizativos y dosimétricos. Estos resultados deben ayudar a establecer prioridades dentro de los programas de garantía de la calidad, basadas en la relación costo-beneficio.

De interés particular es la evaluación realizada recientemente a 61 unidades de mamografía en 11 países de América Latina y el Caribe (18), auspiciada por la OPS y el Colegio Interamericano de Radiología. La calidad de las imágenes se evaluó mediante el maniquí de acreditación del ACR y las mediciones de las dosis aplicadas, mediante dosímetros termoluminiscentes colocados sobre el propio maniquí. En general, $88 \%$ de los servicios cumplieron con los criterios de calidad de la imagen del ACR, aunque en 92\% de los servicios, las dosis se encontraban por debajo de los valores recomendados en las BSS. Estos resultados son similares a los encontrados en Estados Unidos (19). Sin embargo, hay que tener en cuenta que la participación en ese estudio fue totalmente voluntaria y $66 \%$ de los servicios pertenecían a instituciones privadas. Al igual que en el presente estudio, no se halló ninguna correlación entre la calidad de la imagen y las dosis aplicadas.

El hecho de utilizar en este estudio pacientes que fueron atendidos en los departamentos de radiología como parte de su trabajo habitual, si bien complicó la recolección de todos los datos necesarios, permitió contar con una base de datos directamente vinculada a la realidad clínica que se vivía en las instituciones participantes. Por lo general, los estudios disponibles en la literatura se basaron en pacientes seleccionados según criterios de inclusión muy específicos (20). En la presente investigación multicéntrica, la utilización de pacientes sirvió además para que en varios de los departamentos participantes quedaran adoptados métodos de trabajo que ayudarán a mejorar la calidad de las imágenes radiográficas que le llegan al radiólogo informante.

Algunos autores consideran que la calidad de la imagen es el factor determinante para obtener un diagnóstico certero $(21,22)$, lo que quedó confirmado en este estudio. No obstante, la evaluación de la calidad de las imágenes radiográficas se basó en el criterio de los expertos y, aunque a los paneles se les pidió que siguieran los criterios de la Comisión Europea (CE) para la evaluación de la calidad de la imagen (4), no se puede asegurar que la uniformidad entre los diferentes paneles fuera la suficiente como para comparar los resultados de los cinco países. Si bien los expertos fueron respaldados por las sociedades radiológicas nacionales para garantizar 
su competencia profesional, existe un nivel de desarrollo muy diferente en los diversos países participantes. Cuando se han fijado determinados umbrales de calidad para una evaluación, el criterio general de los radiólogos respecto a las imágenes es importante, pero también lo es la calidad de las imágenes que los observadores están acostumbrados a ver (20). Los resultados del presente estudio indican que no es posible comparar la calidad de las imágenes evaluadas por diferentes observadores, aun cuando se utilicen los criterios de la CE (4).

En el presente estudio, los paneles de cada país estaban compuestos por especialistas de reconocido prestigio nacional, lo que permitió a los grupos de investigadores realizar comparaciones entre los diferentes centros participantes, identificar sus propias potencialidades y debilidades y tomar medidas correctoras siempre que fue posible (10, 14, 23). Para poder comparar entre sí los resultados obtenidos en los diferentes países en el futuro, se debe contar con un solo panel de evaluación, con criterios de calidad de la imagen muy bien definidos y entrenados en el uso de esos criterios. Otra debilidad de este estudio fue que muchas de las correlaciones estudiadas carecen de poder estadístico debido al tamaño reducido de la muestra, determinado por las limitaciones financieras.

Las altas dosis de radiación para los estudios de tórax informadas en Bolivia pueden deberse al uso de técnicas de baja tensión, lo que contradice las recomendaciones de la Organización Mundial de la Salud para este tipo de estudio (24). En el caso de Cuba, donde se sobrepasaron los límites de dosis recomendados en las BSS para los estudios de columna vertebral, se utilizaron por lo general valores del producto corriente-tiempo mayores que los habituales (24), posiblemente debido a las dificultades que presentan esos equipos para regular con exactitud y reproducibilidad los tiempos de exposición.

Otras limitaciones de este estudio fueron la poca experiencia de los especialistas participantes en este tipo de investigación, la gran cantidad de variables analizadas y el hecho de que el proyecto no se diseñó inicialmente como un estudio multicéntrico. Además, los instrumentos de medición no fueron los mismos en todos los países, por lo que fue difícil uniformar los protocolos. Para subsanar esta dificultad se compraron algunos otros instrumentos de medición, lo que generó gastos adicionales en cada país y un considerable retraso en el desarrollo de la investigación.

A pesar de esas limitaciones, el presente estudio demostró que la combinación de evaluaciones físicas y clínicas es una buena vía para mejorar el resultado clínico y que este método se puede aplicar en todos los países, independientemente de sus condiciones económicas o del sistema de propiedad de los servicios (estatales, privados o parcialmente privados). La evaluación integral de los departamentos de radiología llevada a cabo en esta investigación permitió establecer, en primer lugar, que se debe prestar mayor atención a la formación continuada de los recursos humanos - especialmente de los técnicos- y que se debe dar prioridad al mantenimiento o reposición de los negatoscopios, las pantallas intensificadoras y las máquinas reveladoras.

El proyecto de investigación tuvo un impacto significativo adicional. En primer lugar, acercó a investigadores de países que se encontraban en diferentes niveles de desarrollo y que se beneficiaron del intercambio de información y experiencias. Otro elemento importante fue la colaboración entre técnicos, físicos médicos y radiólogos en cada país participante, lo que permitió que se reconociera el papel de cada especialista en el mejoramiento de la calidad de los departamentos de radiología. Finalmente, el conocimiento y la experiencia adquiridos por los investigadores de los cinco países participantes deben contribuir a mejorar el estado de los servicios de radiología en esos países y en América Latina y el Caribe.

\section{CONCLUSIONES}

Aunque muchas de las correlaciones estadísticas estudiadas carecen de poder estadístico debido al tamaño reducido de la muestra, este estudio demostró que la buena calidad de la imagen es un elemento crítico para lograr diagnósticos certeros. Se debe hacer hincapié en la formación continuada de los técnicos y en la compra y el mantenimiento de equipos y accesorios, en especial los negatoscopios, las pantallas intensificadoras y las máquinas reveladoras, por la incidencia que tienen en la calidad de las imágenes.

Como resultado de esta investigación, las autoridades nacionales han impuesto o modificado algunas de sus normas: en Argentina, el investigador principal sostuvo conversaciones con los secretarios de salud de varias provincias y le proporcionó detalles acerca de la situación de los servicios al Comité Federal de Salud, lo que llevó a elaborar un plan para solucionar los problemas encontrados; en Bolivia, a partir de los resultados obtenidos se incorporaron criterios específicos para los servicios de radiodiagnóstico como parte del Programa de Acreditación de Hospitales; en Colombia, el Instituto Nacional de Cancerología y la Sociedad de Radiología crearon un programa de garantía de la calidad y se contrató a un físico investigador para ese programa; en Cuba, el Centro de Control Estatal de Equipos Médicos pasó a formar parte del Grupo Nacional Asesor de Radio- 
logía y del Programa de Detección Precoz de Cáncer de Mama, se han realizado varios cursos y talleres y se han elaborado nuevos proyectos de investigación; y en México se revisaron las normas establecidas por el Ministerio de Salud para el control de la calidad de los equipos de rayos $X$.

Agradecimientos. Los autores extienden su agradecimiento a todas las instituciones que participaron en este estudio. De las 182 personas que participaron en él de manera directa, los autores desean agradecer la contribución especial de: Lola Aguilar Serrano, Fernando Barrientos Zamora, Petra E. Beltrán, Lucio Ronald Berdeja Amatller,
Claudia Betancourt, Solángel Bolaños, Elena Carli, Rodolfo de la Nuez Soto, Leonel Gaitán, Waldo García Riverón, Reinaldo Griñán Torres, CarmenRosa Guevara Ferrer, Jorge Kiguen, Roberto Lescano, Miriam Malberti, Hubaldo Martínez, DulceMaría Martínez Pereira, Edgar Medina Delgadillo, Luis-Eduardo Pedroso Mendoza, Rolando Pereiras Costa, Héctor Posso, José Luis Ramírez Arias, Jhonny Rivero Silva, Maytee Robaina García, Jaime Robinson, José L. Rodríguez, Maricel Rodríguez, Miriam Rodríguez Menéndez, César G. Ruiz-Trejo, Genaro-Rodrigo Salinas Mariaca, Margarita Serres Moret, Jorge Skvarca, Roberto Solares Amador, Luis Quevedo Sotolongo, Belkys Vásquez Ríos, Maricela Verdejo-Silva y Yolanda Villaseñor.

\section{REFERENCIAS}

1. Pan American Health Organization. Health in the Americas. 1998 ed. Washington, D.C.: PAHO; 1998. (Scientific Publication 569).

2. Pan American Health Organization. Promoting health in the Americas: annual report of the Director, 2001. Washington, D.C.: PAHO; 2001.

3. Pan American Health Organization Health in the Americas. 2002 ed. Washington, D.C.: PAHO; 2002. (Scientific and Technical Publication 587).

4. European Commission. European guidelines on quality criteria for diagnostic radiographic images. Luxembourg: Luxembourg; 1996. (EUR 161260-EN).

5. Ritter VW, Reid Warren S Jr., Pendergrass EG. Roentgen doses during diagnostic procedures. Radiology. 1952;59: 238-49.

6. U.S. Department of Health, Education and Welfare. Population exposure to X-rays, U.S. 1970. Rockville, MD: DHEW; 1973. (DHEW Publication [FDA] 73-8047).

7. Food and Agriculture Organization, International Atomic Energy Agency, International Labor Organization, Nuclear Agency of the Organization for Economic Co-operation and Development, Pan American Health Organization, World Health Organization. International basic safety standards for protection against ionizing radiation and for the safety of radiation sources. Vienna: IAEA; 1997. (Safety Series No. 115).

8. European Union. Council Directive 97/43/ Euratom, on health protection of individuals against the dangers of ionizing radiation in relation to medical exposure. Official Journal (European Union). 1997;L180:22-7.

9. Sáez Núñez DG, Borroto Valdés $M$, Mesa Hernández M, del Risco Reyna L, Borrás C. Estudio piloto de control de calidad en radiodiagnóstico de hospita- les de Ciudad Habana. Resultados dosimétricos en las radiografías de tórax postero-anterior y columna lumbosacra lateral. Radioprotección.1998;6(17):16-9.

10. Guevara CR, de la Mora R, Fleitas I. Estudio de dosis en exámenes de mamografía en Cuba. Interrelación con la calidad de la imagen obtenida. Memorias del V Congreso Regional de Protección y Seguridad Radiológica; 2001 abril 29-mayo 2; Recife, Brasil. Recife: IRPA; 2001.

11. Guevara CR, de la Mora R, Fleitas I. Resultados obtenidos durante la ejecución de controles de calidad en mamografía. Memorias del Congreso Latinoamericano de Ingeniería Biomédica, mayo 23-25 de 2001, Ciudad de La Habana, Cuba. Ciudad de La Habana: SocBio; 2001.

12. Miranda Cuadros A. Evaluación de la calidad de los servicios de radiodiagnóstico en Bolivia. Informe de investigación del Programa de Subvenciones para la Investigación. Washington, D.C.: OPS; 2002. Disponible en: http://www.paho. org/Spanish /HDP / HDR/BOL-Res3010.doc. Acceso el 26 de marzo de 2006.

13. Fleitas I, Borrás $C$, Brandan ME, Caspani C, Miranda A, de la Mora R, et al. Diagnostic reference levels and quality measurements in five American countries. Proceedings of the World Congress on Medical Physics and Biomedical Engineering, August 24-29 2003, Sydney, Australia. Sidney: IFMBE, IOMP; 2003.

14. Brandan ME, Ruiz Trejo C, Trujillo F, Chávez C, Montes N, Verdejo Silva M, et al. Evaluation of equipment performance, patient dose, imaging quality, and diagnostic coincidence in five Mexico City mammography services. Arch Med Res. 2004;35:24-30.

15. Pan American Health Organization. Development, quality control and radiation protection in radiological services- imaging and radiation therapy. Washington, D.C.: PAHO; 1997.

16. National Council on Radiation Protection and Measurements. Quality assurance for diagnostic imaging. Bethesda, MD: NCRP; 1988. (NCRP Report No. 99).

17. American College of Radiology. Mammography quality control manual. Reston, VA: ACR; 1999.

18. Borrás C, Mota H, Skvarca JJ. Measurements of image quality and dose in 61 mammography units in 11 countries. Proceedings of the 89th Radiological Society of North America Scientific Assembly and Annual Meeting Program, December 1-5 2003, Chicago, IL. Chicago: RSNA; 2003.

19. Suleiman $\mathrm{OH}$, Spelic DC, McCrohan JL, Symonds GR, Houn F. Mammography in the 1990s: The United States and Canada. Radiology. 1999;210:345-51.

20. Lanhede B, Båth M, Kheddache $S$, Sund P, Björneld L, Widell M, et al. The influence of different technique factors on image quality of chest radiographs as evaluated by modified CEC image quality criteria. Br J Radiol. 2002;75: 38-49.

21. Guibelalde del Castillo E. Controles básicos de calidad de imagen con la aplicación de los criterios de la Comunidad Europea y con objetos de test. Memorias del X Congreso Nacional de Física Médica, septiembre de 1995, Salamanca, España. Salamanca: Sociedad Española de Física Médica; 1995.

22. European Commission. CEC quality criteria for Diagnostic Radiographic Images and Patient Exposure Trial. Brussels: CEC; 1990. (EUR $12952 \mathrm{EN}$ ).

23. Brandan ME, Caspani CEM, de la Mora R, Miranda AA, Plazas MC, Borrás C. Evaluation of diagnostic radiology services in five Latin American countries: results for mammography. Proceedings of the International Conference on Radi- 
ological Protection of Patients in Diagnostic and Interventional Radiology, Nuclear Medicine and Radiotherapy, March 26-30 2001, Malaga, Spain. Vienna: IAEA; 2001.
24. World Health Organization. The WHO manual of diagnostic imaging. Radiographic technique and projections. Geneva: WHO; 2003
Manuscrito recibido el 14 de diciembre de 2004. Aceptado para publicación, tras revisión, el 9 de diciembre de 2005

\section{ABSTRACT \\ The quality of radiology services in five Latin American countries}

Objective. To determine the correlation between certain quality indicators for imaging services and the accurate interpretation of radiological exams for four frequent complaints: breast lumps, gastrointestinal discomfort, back pain, and symptoms of tuberculosis.

Methods. Twenty-six radiology services in Argentina, Bolivia, Colombia, Cuba, and Mexico were assessed. The mammography and conventional radiographic/fluoroscopic equipment used in selected services were evaluated utilizing common protocols, data sheets, testing instruments, phantoms, and calibrated dosimetry systems. The studies were performed in medium-complexity facilities. Informed consent was obtained from all patients studied, and the confidentiality of results was guaranteed. The following parameters were documented: type of facility (public vs. private); population covered; patient workload; radiological and image-processing equipment and supplies; education and training of professional and technical staff; quality assurance and preventive maintenance programs, and adherence to radiation safety standards. The performance of $x$-ray units, image receptors and processors; darkroom and image viewing conditions; patient doses and image quality, were determined using standardized parameters in all cases. Independent panels of radiologists, recognized as experts by the local radiological society, assessed the quality of the clinical images obtained and performed a radiological interpretation for each patient using the same films and clinical history available to the institution's imaging physicians. The agreement between the panel of expert's reports and those of local radiologists was taken as an indicator of the radiological diagnostic accuracy.

Results. Analyses were carried out of 366 mammograms, 343 radiological procedures for gastrointestinal complaints, 319 X-rays of the spinal column, and 157 chest radiographs. The agreement between the radiological interpretation of the panel of experts and of the local physician ranged from $70 \%$ to $100 \%$, except in the case of spinal column films in Cuba (57.8\%) and of mammograms in Mexico (33.3\%), which the panel of experts found to be among those having the poorest quality. There was a significant positive correlation between the accuracy of the radiological interpretation and the quality of the radiological images. Image quality showed a positive correlation with the technicians' level of education and training. Studies performed in services that had automatic film processors and that complied with the indicators established for screen-film contact yielded better images and a higher proportion of studies with concordant results. More than $50 \%$ of the viewboxes did not satisfy the quality criteria for luminance and homogeneity.

Conclusions. A good quality image is critical to achieving an accurate diagnosis. Emphasis should be placed on the continuing education of radiology technicians and on the acquisition and maintenance of adequate equipment and accessories, especially viewboxes, intensifying screens, and automatic film processors, given the impact they have on image quality.

Key words Radiology, hospital radiology department, chest radiography, mammography, fluoroscopy, quality control, Latin America. 